\title{
Actualiser la vision des territoires agricoles et ruraux québécois : principales balises pour un exercice incontournable
}

\author{
Gérald Domon et Julie Ruiz \\ Université de Montréal
}

Les chiffres sont bien connus. De 1951 à 2001, le nombre de fermes au Québec est passé de 134000 à 32 000; le nombre de fermes laitières de 96000 à 8600 , et la population agricole de 792000 à $96000^{1}$. S'ils cachent la grande diversité des situations régionales, ces chiffres révèlent néanmoins un fait aujourd'hui incontournable. Ils montrent effectivement l'incapacité du modèle d'agriculture productiviste, qui a guidé les programmes et politiques agricoles au cours des trente dernières années, à assurer le développement viable des communautés rurales.

D'une part, ce modèle a conduit à l'abandon pur et simple de l'agriculture sur de vastes pans du territoire québécois, soit là où les conditions ne se prêtaient pas à une agriculture intensive à grande échelle et à haut rendement. Que ce soit sur le piedmont ou sur les plateaux des Appalaches ou $\mathrm{du}$ Bouclier canadien, la grande majorité des municipalités aura perdu plus de $50 \%$ de ses superficies cultivées entre 1951 et $2001^{2}$. D'autre part, même là où l'agriculture intensive s'est pleinement déployée, soit presque essentiellement dans les Basses Terres du Saint-Laurent, elle n'a pu assurer l'essor économique, social et démographique des collectivités. La diminution spectaculaire du nombre de fermes et de la population agricole y a effectivement remis en cause la viabilité des communautés. Le mémoire déposé par la municipalité de SaintMarcel-de-Richelieu à la Commission sur l'avenir de l'agriculture et de l'agroalimentaire du Québec est, à cet égard, on ne peut plus éloquent. Située sur les meilleures terres agricoles du Québec, cette municipalité a vu sa population passer de 900 à 580 personnes. Aussi paradoxal que ce soit, tout en étant proche de centres urbains d'importance, Saint-Marcel-deRichelieu « a commencé depuis plusieurs années déjà à vivre des réalités sociales et économiques qui se rapprochent étrangement de celles trouvées dans certaines petites municipalités des régions dites périphériques $»^{3}$.

Dans la mesure où non seulement la viabilité de ce modèle agricole est visée, mais également celle de l'ensemble des communautés rurales, l'évolution récente de l'agriculture force à reconsidérer les rapports entre l'agricole et le rural. Elle force notamment à actualiser notre vision de l'un et de l'autre et à modifier en conséquence nos approches d'aménagement. Puisque espace rural et espace agricole se sont historiquement chevauchés au point de se confondre, il s'agit là d'un défi considérable. Celui-ci n'est toutefois pas unique au Québec. Aussi, plusieurs pays occidentaux sont-ils déjà fortement engagés dans une révision profonde des liens qui unissent l'agricole et le rural. De ces expériences, un certain nombre d'enseignements ressortent comme incontournables. Ils fournissent les principales balises en vue de l'actualisation des visions qui s'impose au Québec.

\section{La multifonctionnalité des territoires}

Que la population agricole, devenue partout minoritaire au sein de la population rurale, ne puisse à elle seule assurer la viabilité des communautés, cela est de plus en plus admis. Plus encore, on ne met plus en doute que la production de biens alimentaires n'est qu'une des fonctions que peut assurer l'espace rural. Dans ce nouveau contexte dit «post-productiviste ${ }^{4}$, les territoires ruraux doivent répondre non seulement aux attentes liées à la production (biens agroalimentaires), mais aussi à celles liées à la consommation (tourisme, villégiature, habitation) et à la conservation (patrimoine naturel et culturel) de l'espace rural. Aussi, la viabilité des communautés est-elle aujour- 
d'hui étroitement liée à la multifonctionnalité du territoire, c'est-à-dire à la présence et au maintien de différentes fonctions au sein d'un territoire donné.

$\mathrm{Si}$, pour certains, la multifonctionnalité peut sembler une caractéristique intrinsèque ou potentiellement intrinsèque à tout territoire, sa reconnaissance pose dans les faits un défi singulier pour le Québec. Comme nous le rappellent invariablement les études historiques régionales et les travaux sur la reconstitution de l'évolution des paysages agroforestiers ${ }^{5}$, c'est effectivement l'exploitation des ressources, forestière puis agricole, qui, de la plaine montérégienne à Charlevoix, en passant par les Cantons-de-l'Est, l'Abitibi, la Matapédia ou la Côte-du-Sud, est à l'origine de la colonisation et du développement du territoire québécois. Cette tradition de colonisation et de développement basée sur l'exploitation des ressources a marqué et continue de marquer profondément notre vision du territoire tout autant que nos programmes et politiques d'aménagement et de mise en valeur. Aussi, reconnaître que les territoires agricoles de la Montérégie, de Lanaudière, des Cantons-de-l'Est ou du Centre-du-Québec ne sont plus que des espaces de production de biens agroalimentaires, mais qu'ils sont également des cadres de vie, des espaces de récréation et de tourisme, des réserves de patrimoine naturel et culturel, demeure dans les faits un défi aussi considérable qu'incontournable. Bien plus que des ajustements marginaux, cette reconnaissance commande une vision du territoire qui tranche singulièrement avec le passé.

\section{Seule une forme de découplage des aides gouvernementales au volume de biens agricoles produits est aujourd'hui à même de maintenir une agriculture vivante et diversifiée sur le territoire québécois.}

\section{Le découplage des aides gouvernementales et de la production agricole}

Face aux risques de surproduction, à l'ouverture croissante des marchés et à la reconnaissance de la multifonctionnalité des territoires, une seconde tendance lourde consiste à dissocier les aides gouvernementales de la production agricole. Ainsi, ces aides ne sont plus uniquement tributaires des superficies mises en culture ou des volumes récoltés, mais aussi des autres services rendus à la collectivité : pratiques soucieuses du maintien de la qualité des eaux, de la protection de la biodiversité, de l'entretien du patrimoine bâti (ex. : granges historiques) et des paysages.

En France, par exemple, les Contrats territoriaux d'exploitation (CTE; remplacés successivement depuis lors par des Contrats d'agriculture durable puis par des mesures agro-environnementales territorialisées) ont constitué la mesure phare de mise en œuvre de la multifonctionnalité agricole à la suite de la réforme de la Loi d'orientation agricole en 1999. Ces contrats devaient reposer sur un projet global pour l'exploitation comprenant un volet socio-économique et un volet environnemental et territorial. L'exploitant devait proposer un projet comprenant des actions allant au-delà des lois et des règlements en vigueur; cet effort additionnel lui valant une compensation financière selon les règles européennes. Aussi, les candidats aux CTE s'engageaient-ils à entreprendre des actions en faveur de la protection de la biodiversité, de l'entretien ou de la restauration des paysages, de l'amélioration de la qualité de l'eau ou de la qualité des produits.

À l'évidence, une approche comme celle des CTE en appelle d'une adaptation au contexte québécois, notamment en regard de la lourdeur de son opérationnalisation. Cela dit, il paraît acquis que seule une forme de découplage des aides gouvernementales au volume de biens agricoles produits est aujourd'hui à même de maintenir une agriculture vivante et diversifiée sur le territoire québécois.

\section{Une approche territoriale}

Puisque le territoire n'est plus le support d'une fonction unique, les approches sectorielles tendent aujourd'hui fortement à laisser place à des approches territoriales. C'est effectivement sur la base d'un diagnostic territorial prenant en compte non seulement les objectifs nationaux de production, mais également l'ensemble des ressources, des activités et des problématiques spécifiques à chaque région, voire à chaque groupe de municipalités, que tendent à être définis les programmes et les aides gouvernementales.

En Grande-Bretagne, par exemple, le «UK Agriculture Act» établissait dès 1987 des « Environmentally Sensitive Areas » (ESA) qui permettaient d'instaurer une série de mesures propres aux caractéristiques et 
problématiques des territoires ciblés. Considérée comme la principale mesure agro-environnementale britannique, l'approche sur laquelle reposent les ESA tente de combiner des actions en faveur des paysages, de l'accès à la campagne et de la protection de la nature et des sites historiques. Les agriculteurs peuvent être ainsi indemnisés pour la mise en place de certaines pratiques (maintien des murets de pierre, implantation de haies, etc.) et l'arrêt d'autres (drainage des terres, utilisation de pesticides, etc.) qui tiennent compte des spécificités et des problématiques territoriales de chaque ESA, l'adhésion de chaque exploitant reposant sur une base volontaire.

Par delà des politiques sectorielles agricoles, forestières, touristiques ou autres, l'approche privilégiée par les ESA témoigne d'une volonté de définir de véritables politiques d'aménagement des territoires. Compte tenu de la grande diversité géographique, culturelle, économique de ses régions, le Québec gagnerait, de toute évidence, à s'inspirer de telles approches. Sur ce plan, et quoique leur mandat demeure fort limité, les organismes de bassin versant mis en place dans différentes régions du Québec pourraient constituer de véritables laboratoires; du moins au sein des zones d'intensification agricole.

\section{La nature et le paysage comme leviers de développement}

Alors qu'au Québec, la question de l'environnement, des milieux naturels et des paysages a toujours été et continue d'être essentiellement perçue comme une série de contraintes au développement, un nombre croissant de pays y voient un levier de développement économique et social. De la France à la GrandeBretagne, en passant par les Pays-Bas, l'Allemagne, l'Australie et plusieurs États américains, les milieux naturels et la qualité des paysages sont vus comme des atouts importants répondant à l'intérêt croissant des individus et des entreprises privées envers la qualité des milieux de vie et le développement durable.

Sur ce plan, l'exemple des Pays-Bas parait tout particulièrement intéressant. Alors que, pour des raisons géographiques évidentes, on a historiquement cherché à contrôler et à rendre productive chaque parcelle à des fins agricoles, la vision du territoire y est aujourd'hui en profonde transformation. Ainsi, se démarquant nettement des visées de modernisation, de rationalisation et d'intensification qui caractérisaient le fonctionnalisme d'après-guerre ${ }^{6}$, ces politiques et législations cherchent aujourd'hui non seulement à établir un certain équilibre entre production et conservation, mais aussi à prendre explicitement appui sur la nature et les dimensions qualitatives de l'espace et ce, de manière à assurer le maintien du développement économique et à répondre à la demande sociale croissante. C'est ainsi que des projets comme ceux de l'île de Tiengemeten ${ }^{7}$ revisitent la vocation agricole de vastes territoires à des fins de renaturalisation. Aussi est-il pour le moins paradoxal, alors que d'autres pays investissent des millions d'euros afin de créer de toutes pièces des milieux « naturels » pour répondre à la nouvelle demande sociale, que le Québec, si riche en milieux naturels et en paysages, n'a toujours pas appris à saisir ces opportunités et continue à entretenir une vision monofonctionnelle et productiviste du territoire et, du coup, à dilapider ce riche patrimoine.

\section{Les milieux naturels et la qualité des paysages sont vus comme des atouts importants.}

Alors que plusieurs pays s'emploient depuis plus d'une décennie déjà à réviser leurs programmes et leurs politiques afin de s'ajuster aux nouvelles réalités qui les sous-tendent, le Québec tarde encore à simplement reconnaître ces dernières. Ce rapide survol des tendances lourdes qui marquent les approches au rural et à l'agricole dans d'autres pays met d'autant plus en évidence l'écart toujours croissant entre les programmes et politiques mis de l'avant par les instances centrales et les démarches initiées par les individus au niveau local. Ces démarches témoignent effectivement non seulement d'une grande vitalité, mais aussi d'une inventivité qui pourrait bien être spécifique au Québec et sur laquelle il nous faudrait miser.

\section{Une inventivité à mettre à profit}

En marge des tendances à la spécialisation, à l'intensification et à la concentration des pratiques agricoles, un nombre considérable d'initiatives alternatives ont émergé ces dernières années. Au Québec, ces initiatives alternatives ont conduit tantôt à l'invention de nouveaux produits (fromages, cidres de glace, boissons artisanales, etc.) ou de pratiques permettant des productions nouvelles sur le territoire (production de vins, de lavande, etc.), tantôt encore à la réinvention de pratiques anciennes (élevages d'agneaux, de chè- 
vres, etc.) à des fins nouvelles (vente à la ferme, production de produits dérivés, etc.). Prenant appui sur les demandes croissantes des consommateurs pour les aliments de qualité, l'environnement, le bien-être des animaux ou les produits locaux, ces initiatives participent à l'émergence de nouvelles zones de production $^{8}$.

De l'introduction de l'élevage de bœufs Highland à Bolton-Ouest à l'agneau de Charlevoix en passant par le Cerf de Boileau; des vergers de poires de Shefford aux vergers de prunes de Saint-André ou de cerises à Charrette en Mauricie; du fromage de Suisses brunes en Montérégie à celui de vaches Canadiennes aux Îles-de-la-Madeleine; du vin de l'Orpailleur au cidre de glace de Hemmingford à l'hydromel de FermeNeuve ou à l'alcool aux petits fruits de Bellechasse, l'invention ou la réinvention de produits et de pratiques agricoles ont ces dernières années permis d'explorer d'autres façons de concevoir et d'occuper le territoire. À cet égard, l'analyse, même sommaire, de ces initiatives met en évidence le fait qu'au Québec, les efforts déployés pour actualiser la vision du rural et de l'agricole sont à la fois réels, nombreux et très diversifiés. Fait significatif, dans toutes les initiatives relevées, la production est couplée avec une autre forme d'activité : activité agrotouristique (L'Orpailleur), activité de transformation (Cidre de glace) ou activité de vente directe à la ferme (bœuf Highland de la famille Badger) et s'inscrit donc pleinement dans une perspective de multifonctionnalité du territoire. Enfin, et de toute évidence, ces efforts ne sont pas la résultante d'initiatives de l'État, mais bel et bien d'initiatives locales, voire individuelles. Enfin encore, tout aussi nombreuses et variées qu'elles soient, la viabilité de ces initiatives sur le moyen et le long terme reste à mesurer; cette viabilité étant à l'évidence tributaire de deux principaux facteurs, à savoir : a) la capacité à ajuster les modes de distribution de manière à s'assurer que les producteurs aient un véritable accès au marché; b) la reconnaissance explicite de l'importance de ces initiatives et, conséquemment, de leur prise en compte plus formelle par les programmes de soutien aux activités agricoles.

En définitive, tant en regard de la reconnaissance effective de la multifonctionnalité du territoire, de la territorialisation des aides et de leur découplage de la production, ou encore de la mise en valeur des dimensions qualitatives du territoire, les expériences menées à l'étranger au cours des deux dernières décen- nies plaident pour l'abandon de politiques nationales appliquées de manière uniforme à l'ensemble du territoire. Elles révèlent du coup la nécessité de privilégier des approches flexibles permettant d'identifier et d'appliquer des mesures spécifiques aptes à tenir compte de caractéristiques locales et ce, tant au plan biophysique que social. Visant à appuyer et à stimuler les initiatives locales, les mesures d'aide au développement de produits de spécialité ${ }^{9}$, tout comme celles visant la mise en place de laboratoires ruraux ${ }^{10}$ annoncées à l'été 2007 , semblent s'inscrire dans cette perspective nouvelle. Elles pourraient marquer l'amorce de cette incontournable actualisation des visions de l'agricole et du rural au Québec.

\section{Dans toutes les initiatives relevées, la production est couplée avec une autre forme d'activité.}

\section{Notes et références}

1 Statistique Canada 1951, 2001.

2 Ruiz, J. et G. Domon (2005). « Les paysages de l'agriculture en mutation », dans P. Poullaouec-Gonidec, G. Domon et S. Paquette (dir.), Paysages en perspective, Presses de l'université de Montréal, série « Paysages », Montréal, p. $47-97$

3 Municipalité de Saint-Marcel-de-Richelieu (2007). «L'avenir des petites municipalités rurales dans les milieux d'agriculture intensive au Québec, le cas de Saint-Marcelde-Richelieu, Municipalité régionale de Comté des Maskoutains », mémoire déposé à la Commission sur l'avenir de l'agriculture et de l'agroalimentaire québécois, p. 22.

4 Bowler I.R., et B.W. Ilbery (1999). « Agricultural Landuse and Landscape Change under the Post-productivist Transition - Examples from the United Kingdom », dans R. Krönert J. Baudry, I.R. Bowler et al. (dir.), Land-use Changes and their Environmental Impact in Rural Areas in Europe, Man and the Biosphere Series, UNESCO and The Parthenon Publishing Group, Paris, 121-139.

5 Domon G., et A. Bouchard (2007). « The Landscape History of Godmanchester (Québec, Canada), Two Centuries of Shifting Relationships between Anthropic and Biophysical Factors », Landscape Ecology, n 22, p. 1201-1214.

6 Doevendans, K., H. Lörzing et A. Schram (2007). « From Modernist Landscapes to New Nature : Planning of Rural Utopias in the Netherlands », Landscape Research, vol. 32, p. 333-354.

7 http://www.tiengemeten.com/ 
8 Ilbery, B. et M. Kneafsey. (1998). «Product and Place : Promoting Quality Products and Services in the Lagging Regions of the European Union ", European Urban and Regional Studies, ${ }^{\circ}$ 5, p. 330-341.

$9 \quad$ Ministère des Affaires municipales et des Régions (2007). Mesures pour stimuler le développement de produits de spécialité, politique nationale de la ruralité 2007-2014, Gouvernement du Québec, Québec.

10 Ministère des Affaires municipales et des Régions (2006). Politique nationale de la ruralité 2007-2014, Gouvernement du Québec, Québec. 


\section{Publicité}

\section{Les études de cycles supérieurs}

